\title{
On digital approximations for elliptic boundary value problems
}

Cite as: AIP Conference Proceedings 2293, 090008 (2020); https://doi.org/10.1063/5.0026573

Published Online: 25 November 2020

\section{Oksana Tarasova, and Vladimir Vasilyev}
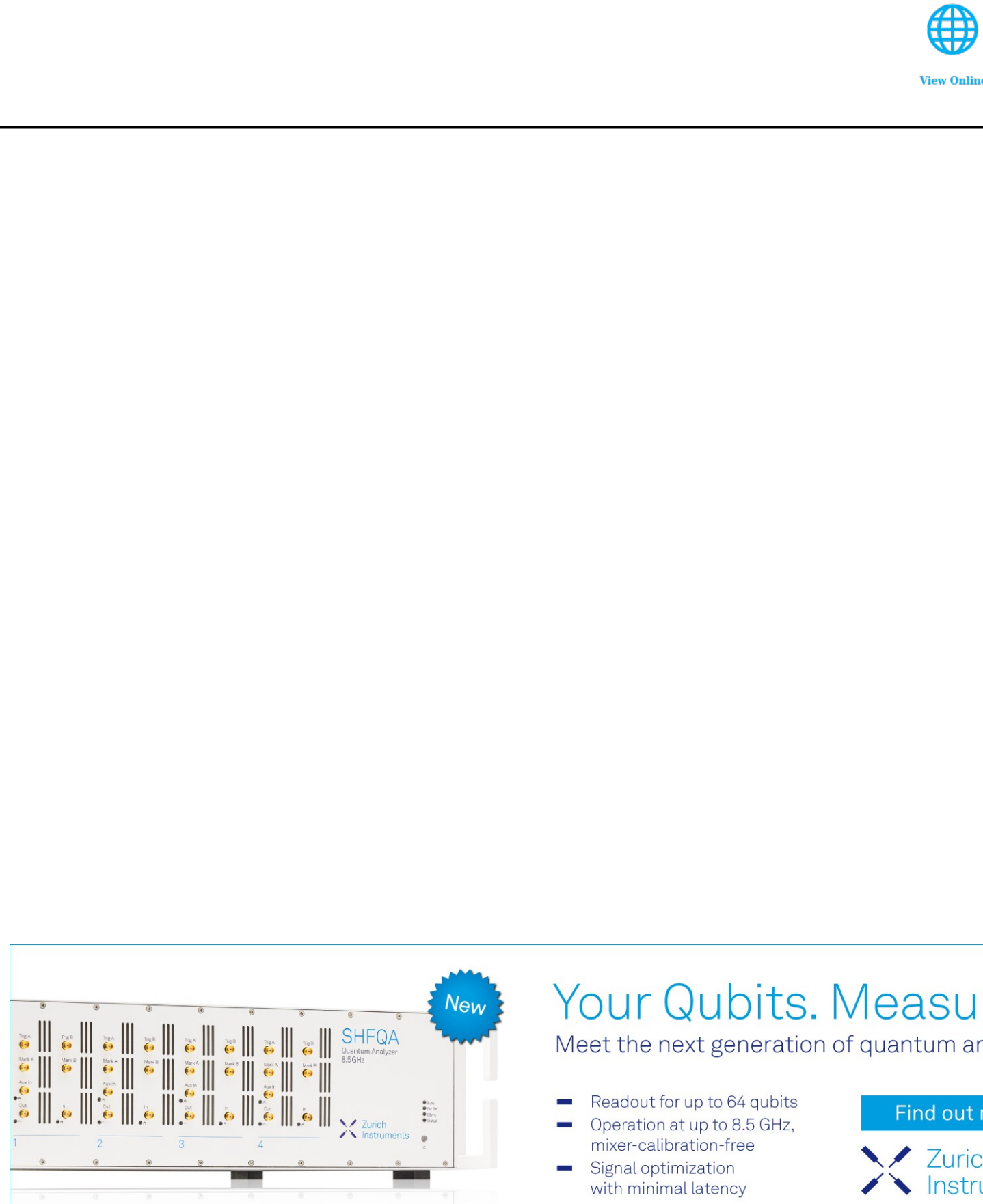

\section{Your Qubits. Measured.}

Meet the next generation of quantum analyzers

- Readout for up to 64 qubits

- Operation at up to $8.5 \mathrm{GHz}$. mixer-calibration-free

- Signal optimization with minimal latency 


\title{
On Digital Approximations for Elliptic Boundary Value Problems
}

\author{
Oksana Tarasova ${ }^{1, \mathrm{~b})}$ and Vladimir Vasilyev ${ }^{1, \mathrm{a})}$ \\ ${ }^{1}$ Chair of Differential Equations, Belgorod State National Research University, Pobedy street 85, Belgorod 309015, \\ Russia

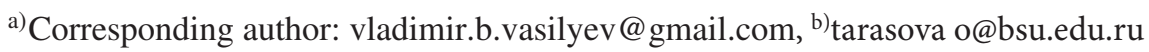

\begin{abstract}
We study certain discrete boundary value problems for discrete analogue of model elliptic pseudo-differential operator in discrete Sobolev-Slobodetskii spaces. Existence and uniqueness theorems are proved and a comparison between discrete and continuous solutions are given.
\end{abstract}

\section{Introduction}

As a rule the classical pseudo-differential operator in Euclidean space $\mathbb{R}^{m}$ is defined by the formula

$$
(A u)(x)=\int_{\mathbb{R}^{m}} \tilde{A}(x, \xi) e^{-i x \cdot \xi} \tilde{u}(\xi) d \xi,
$$

where the sign $\sim$ over a function denotes its Fourier transform

$$
\tilde{u}(\xi)=\int_{\mathbb{R}^{m}} u(x) e^{i x \cdot \xi} d x,
$$

and the function $\tilde{A}(x, \xi)$ is called a symbol of a pseudo-differential operator $A$.

Our main goal here is describing a periodic variant of this definition and studying its certain properties related to solvability of corresponding equations in canonical domains of an Euclidean space. Some important results are related to a comparison of discrete and continuous solutions. We try to preserve maximal correspondence for discrete and continuous cases under digitization, it permits to find more appropriate constructions.

This problem is very large and in our opinion it should include the following aspects according to a lot of physical and technical applications of such operators and related equations, for example, finite and infinite discrete Fourier transform as a natural technique for such equations; choice of appropriate discrete functional spaces; studying solvability for infinite discrete equations; studying solvability of approximating finite discrete equations; a comparison between continuous and infinite discrete equations; a comparison between infinite discrete and finite discrete equations.

This is not completed list of questions for studying which we intend to consider. some results in this direction were obtained for simplest pseudo-differential operators (Calderon-Zygmund operators) and corresponding equations. Also certain results are related to approximate solutions.

There are few variants of the theory of discrete boundary value problems, but these theories are related especially to partial differential operators and do not use the harmonic analysis technique. Since the classical theory of pseudo-differential operators is based on the Fourier transform we will use the discrete Fourier transform and discrete analogue of pseudo-differential operators which will include discrete analogues of partial differential and some integral convolution operators. 


\section{Constructions}

Let $\mathbb{Z}^{m}$ be the integer lattice in $\mathbb{R}^{m}, \mathbb{T}^{m}=[-\pi, \pi]^{m}$ be m-dimensional cube, $h>0, \hbar=h^{-1}$. For functions $u_{d}$ of a discrete variable defined on $h \mathbb{Z}^{m}$, we introduce the discrete Fourier transform

$$
\tilde{u}_{d}(\xi) \equiv\left(F_{d} u_{d}\right)(\xi)=\sum_{x \in h \mathbb{Z}^{m}} u_{d}(x) e^{i x \cdot \xi} h^{m}, \quad, \xi \in \hbar \mathbb{T}^{m}
$$

Let $A_{d}(\xi)$ be a the periodic function in $\mathbb{R}^{m}$ with the main cube of periods $\hbar \mathbb{T}^{m}$. The operator of the type

$$
\left(A_{d} u_{d}\right)(x)=\frac{1}{(2 \pi)^{m}} \sum_{y \in h \mathbb{Z}^{m}} \int_{\hbar \mathbb{T}^{m}} A_{d}(\xi) e^{i(y-x) \cdot \xi} u_{d}(y) h^{m} d \xi, \quad x \in D_{d},
$$

$D_{d}=D \cap h \mathbb{Z}^{m}, D \subset \mathbb{R}^{m}$, we call a discrete pseudo-differential operator in a discrete domain $D_{d}$.

This operator is associated with the equation

$$
\left(A_{d} u_{d}\right)(\tilde{x})=v_{d}(\tilde{x}), \quad \tilde{x} \in D_{d},
$$

where $v_{d}$ is given function in $D_{d}$.

One of the main goals is describing the conditions for unique solvability of the equation in appropriate functional spaces in the discrete domain $D_{d}$ and application of the obtained results to the construction of suitable approximations of classical pseudo-differential equations. The main difficulty is that when a domain $D$ is a part of $\mathbb{R}^{m}$ (for example, a half-space or a cone), the standard ellipticity condition of the symbols $A(\xi)$ is no longer sufficient.

\section{Discrete Boundary Value Problems}

We will study the equation (1) in the discrete domain $D_{d} \equiv D \cap h \mathbb{Z}^{m}$ and will seek a solution $u_{d} \in H^{s}\left(D_{d}\right), v_{d} \in$ $H_{0}^{s-\alpha}\left(D_{d}\right)$. Everywhere here $D=\mathbb{R}_{+}^{m}$. Solvability of the equation depends on special number $\kappa$ which is called an index of periodic factorization $\tilde{A}_{d}(\xi)[1,2]$.

We have non-uniqueness of a solution for the equation (4) for the case $\kappa-s=n+\delta, n \in \mathbb{N},|\delta|<1 / 2$. We consider here the case $n=1$. To obtain the unique solution one needs some additional conditions. Discrete analogues of Dirichlet or Neumann conditions give a very simple case. We will consider here the discrete Dirichlet condition.

$$
\left.u_{d}\right|_{\tilde{x}_{m}=0}=g_{d}\left(\tilde{x}^{\prime}\right),
$$

where $g_{d}$ is a given function of a discrete variable in the discrete hyper-plane $\mathbb{Z}^{m-1}$.

To obtain some comparison between discrete and continuous solutions we will remind how the continuous solution looks. The continuous analogue of the discrete boundary value problem is the following

$$
\begin{gathered}
(A u)(x)=0, \quad x \in \mathbb{R}_{+}^{m}, \\
u\left(x^{\prime}, 0\right)=g\left(x^{\prime}\right), \quad x^{\prime} \in \mathbb{R}^{m-1},
\end{gathered}
$$

where $A$ is elliptic pseudo-differential operator with symbol $A(\xi)$ satisfying the condition

$$
c_{1}(1+|\xi|)^{\alpha} \leq|A(\xi)| \leq c_{2}(1+|\xi|)^{\alpha}, \quad \alpha \in \mathbb{R}
$$

with a positive constant $c_{1}, c_{2}$.

If the index of factorization equals $\kappa$ and $\kappa-s=1+\delta,|\delta|<1 / 2$ then the unique solution for the problem is constructed by the similar formula

$$
\tilde{u}(\xi)=b^{-1}\left(\xi^{\prime}\right) \tilde{g}\left(\xi^{\prime}\right) A_{+}^{-1}\left(\xi^{\prime}, \xi_{m}\right), \quad b\left(\xi^{\prime}\right)=\int_{-\infty}^{+\infty} A_{+}^{-1}\left(\xi^{\prime}, \xi_{m}\right) d \xi_{m}
$$

assuming that $b\left(\xi^{\prime}\right) \neq 0, \forall \xi^{\prime} \in \mathbb{R}^{m-1}$. Let us note that this is simplest variant of Shapiro-Lopatinskii condition. 
We have the following discrete solution

$$
\tilde{u}_{d}(\xi)=b_{d}^{-1}\left(\xi^{\prime}\right) \tilde{g}_{d}\left(\xi^{\prime}\right) A_{d,+}^{-1}\left(\xi^{\prime}, \xi_{m}\right), \quad b_{d}\left(\xi^{\prime}\right)=\int_{-\hbar \pi}^{+\hbar \pi} A_{d,+}^{-1}\left(\xi^{\prime}, \xi_{m}\right) d \xi_{m},
$$

if

$$
\inf _{\xi^{\prime} \in \mathbb{T}^{m-1}}\left|b_{d}\left(\xi^{\prime}\right)\right|>0
$$

We will assume the condition (5) is satisfied for the discrete symbol $A_{d}(\xi)$..

Further we choose special approximations. We take $g_{d}=Q_{h} g$, where $Q_{h}$ is special projector on discrete functional space [3] and $A_{d, \pm}\left(\xi^{\prime}, \xi_{m}\right)$ we take as restrictions of $A_{ \pm}\left(\xi^{\prime}, \xi_{m}\right)$ on $\hbar \mathbb{T}^{m}$. Then the periodic symbol

$$
A_{d}(\xi)=A_{d,+}\left(\xi^{\prime}, \xi_{m}\right) A_{d,-}\left(\xi^{\prime}, \xi_{m}\right)
$$

satisfies all conditions of periodic factorization with the same index æ. Moreover, $\tilde{g}_{d}\left(\xi^{\prime}\right)$ and $A_{d,+}\left(\xi^{\prime}, \xi_{m}\right)$ coincide with $\tilde{g}\left(\xi^{\prime}\right)$ and $A_{+}\left(\xi^{\prime}, \xi_{m}\right)$ respectively on $\hbar \mathbb{T}^{m}$.

\section{Main Results}

Theorem 1. Discrete boundary value problem (1),(2) is uniquely solvable in the space $H^{s}\left(h \mathbb{Z}_{+}^{m}\right)$ for arbitrary righthand side $v_{d} \in H_{0}^{s-\alpha}\left(h \mathbb{Z}_{+}^{m}\right)$ and arbitrary boundary function $g_{d} \in H^{s-1 / 2}\left(h \mathbb{Z}^{m-1}\right)$.

Theorem 2. Let $\kappa>1$. If $\tilde{g}(\xi)$ is a bounded function then a comparison between solutions of problems (1),(2) and (3),(4) is given in the following way

$$
\left|\tilde{u}(\xi)-\tilde{u}_{d}(\xi)\right| \leq C h^{\kappa-1}, \quad \xi \in \hbar \mathbb{T}^{m}
$$

Further we consider the following discrete boundary value problem for the equation (1) with the discrete boundary condition

$$
\int_{-\hbar \pi}^{\hbar \pi} \hbar\left(e^{-i h \xi_{m}}-1\right) \tilde{u}_{d}\left(\xi^{\prime}, \xi_{m}\right) d \xi_{m}=\tilde{g}_{d}\left(\xi^{\prime}\right), \quad \xi^{\prime} \in \hbar \mathbb{T}^{m-1},
$$

where $\tilde{g}_{d}\left(\xi^{\prime}\right)$ is the given function, and its continuous analogue

$$
\begin{gathered}
(A u)(x)=0, \quad x \in \mathbb{R}_{+}^{m}, \\
\left.\frac{\partial u}{\partial x_{m}}\right|_{x_{m}=0}=g\left(x^{\prime}\right), \quad x^{\prime} \in \mathbb{R}^{m-1} .
\end{gathered}
$$

It is well known that the boundary value problem (7),(8) is uniquely solvable in Sobolev-Slobodetsky spaces $H^{s}\left(\mathbb{R}_{+}^{m}\right)$ for arbitrary any right-hand side $g \in H^{s-\frac{3}{2}}\left(\mathbb{R}^{m-1}\right)$ under some restrictions on the factorization index $\kappa$ of the symbol $A(\xi)$, namely $\kappa-s=1+\delta,|\delta|<1 / 2$.

Theorem 3. The elliptic boundary value problem (1),(6) under $v_{d} \equiv 0$ is uniquely solvable in the space $H^{s}\left(D_{d}\right)$ for arbitrary right-hand side $g_{d} \in H^{s-3 / 2}\left(h \mathbb{Z}^{m-1}\right)$.

If $s \geq 1$ then the a priori estimate

$$
\left\|u_{d}\right\|_{s} \leq C\left\|g_{d}\right\|_{s-3 / 2}
$$

holds with constant $C$ non-depending on $h$.

Theorem 4. If $g \in H^{s-3 / 2}\left(h \mathbb{Z}^{m-1}\right), \mathfrak{}>2, \beta \leq 5 / 2+\delta$, then a comparison between solutions of problems (1),(6) and (7),(8) is given by the estimate

$$
\left\|Q_{h} u-u_{d}\right\|_{\beta} \leq C h^{\mathfrak{x}-2}\left\|g_{d}\right\|_{s}
$$

with constant $C$ non-depending on $h$. 


\section{Conclusions}

This paper is one of first steps for studying discrete boundary value problems and their connections with classical theory of boundary value problems for elliptic pseudo-differential equations. We intend to study more general situations in forthcoming papers and to obtain approximation estimates for comparison of discrete and continuous solutions

\section{ACKNOWLEDGMENTS}

This work was supported by the State contract of the Russian Ministry of Education and Science (contract No 1.7311.2017/8.9).

\section{REFERENCES}

[1] A. V. Vasilyev and V. B. Vasilyev, Math. Model. Anal. 23, 492-506 (2018).

[2] A. V. Vasilyev and V. B. Vasilyev, Tatra Mt. Math. Publ. 71, 195-212 (2018).

[3] O. A. Tarasova and V. B. Vasilyev, 4Open 2, 1-7 (2019). 